\title{
Placement and fixation of the endotracheal tube in trauma patients
}

\author{
Espen Cramer Korsvold \\ From Danish Society for Emergency Medicine: Research Symposium 2010 \\ Roskilde, Denmark. 20-21 May 2010
}

\section{Background}

To investigate the incidence of bronchial intubation in trauma patients intubated prehospital or in-hospital, in connection with receiving and treating of trauma patients at the Trauma Center at Aarhus University Hospital.

To investigate whether the materials currently used for fixation of the tracheal tube ensures secure fixation of the tracheal tube.

To investigate whether the method of fixation influences the likelihood of bronchial intubation.

\section{Methods}

Prospective registration of the incidence of bronchial intubation in 100 endotracheal intubated trauma patients admitted to the Trauma Center at Aarhus University Hospital from January 2008 to January 2009. Additionally, the method of controlling tube placement, the fixation method used and condition of the patient's face were recorded.

\section{Results}

No oesophageal intubations were documented during the project period (100 patients)!

A total of 17 bronchial intubations occurred in 13 patients (some patients were bronchial intubated twice or more)

Bronchial intubations were diagnosed using chest $\mathrm{x}$-ray in 10 patients, by lung stethoscopy in 5 patients and by $\mathrm{CT}$ scan in 2 patients.

86 satisfactory fixated tubes were registered.

14 tubes were recorded as unsatisfactorily fixated: 9 fixations were reinforced and 5 fixation materials had to be replaced.

Correspondence: espen@korsvold.dk

CRNA, Aarhus University Hospital Department of Orthopaedic anaesthesia and Trauma Center Aarhus, Denmark
Nine of these patients had facial trauma including blood and facial hair which may have influenced the quality of the fixation

3 of the broncially placed tubes were recorded as not satisfactory fixated.

\section{Conclusion}

The number of bronchial intubations should be minimised, and a misplaced endotracheal tube must be diagnosed as early as possible.

This study shows a prevalence of broncial tube placement at $17 \%$, therefore it would be appropriate to focus attention on the control of tube placement as quickly as possible after the patient's arrival or intubation in hospital.

A chest X-ray should be taken as early as possible.

The number of insufficient tube fixations has locally led to use of the Thomas(TM) endotracheal tube holder, which has been tested and now is the standard fixation material in our Trauma Center.

Published: 17 September 2010

doi:10.1186/1757-7241-18-S1-P25

Cite this article as: Korsvold: Placement and fixation of the

endotracheal tube in trauma patients. Scandinavian Journal of Trauma,

Resuscitation and Emergency Medicine 2010 18(Suppl 1):P25. 